\title{
The state of the art in breast imaging using the Twente Photoacoustic Mammoscope: results from 31 measurements on malignancies
}

\author{
Michelle Heijblom ${ }^{1,2}$ - Daniele Piras ${ }^{1}$ - Frank M. van den $\operatorname{Engh}^{2} \cdot$ Margreet van $^{2}$ \\ der Schaaf $^{2}$ - Joost M. Klaase ${ }^{2} \cdot$ Wiendelt Steenbergen $^{1} \cdot$ Srirang Manohar $^{1}$
}

Received: 13 June 2015 /Revised: 9 January 2016 / Accepted: 22 January 2016 / Published online: 5 March 2016

(C) The Author(s) 2016. This article is published with open access at Springerlink.com

\begin{abstract}
Objectives Photoacoustic mammography is potentially an ideal technique, however, the amount of patient data is limited. To further our understanding of the in vivo performance of the method and to guide further research and development, we imaged 33 breast malignancies using the research system - the Twente Photoacoustic Mammoscope (PAM).

Methods Thirty-one patients participated in this retrospective, observational study. The study and informed consent procedure were approved by the local ethics committee. PAM uses 1,064 nm light for excitation with a planar, 588-element, 1$\mathrm{MHz}$ ultrasound array for detection. Photoacoustic lesion visibility and appearance were compared with conventional imaging (x-ray mammography and ultrasonography) findings, histopathology and patient demographics.

Results Of 33 malignancies 32 were visualized with high contrast and good co-localization with conventional imaging. The contrast of the detected malignancies was independent of radiographic breast density, and size estimation was reasonably good with an average $28 \%$ deviation from histology. However, the presence of contrast areas outside the malignant region is suggestive for low specificity of the current system. Statistical analyses did not reveal any further relationship between PAM results and patient demographics nor lesion characteristics.
\end{abstract}

Srirang Manohar

s.manohar@utwente.nl

1 Biomedical Photonic Imaging Group, MIRA Institute for Biomedical Technology and Technical Medicine, University of Twente, P.O. Box 217, 7500 AE Enschede, The Netherlands

2 Center for Breast Care, Medisch Spectrum Twente, P.O. Box 50.000, 7500 KA Enschede, The Netherlands
Conclusions The results confirm the high potential of photoacoustic mammography in future breast care.

Key Points

- Photoacoustic breast imaging visualizes malignancies with high imaging contrast.

- Photoacoustic lesion contrast is independent of the mammographically estimated breast density.

- No clear relationship exists between photoacoustic characteristics and lesion type, grade, etc.

- Photoacoustic specificity to breast cancer from some cases is not yet optimal.

Keywords Photoacoustic techniques - Breast neoplasms · Diagnostic imaging $\cdot$ Pulsed lasers $\cdot$ Ultrasound

\section{Introduction}

With breast cancer being the most common cause of cancer and one of the major causes of cancer death among women [1, 2], there is a continuous search for improved methods for noninvasively imaging the breast and its abnormalities. Such methods either focus on developments in existing techniques [3-5] or investigate new approaches [6-8], not only for improvements in performance, such as sensitivity and/or specificity, but also for improvements in widespread applicability, patient comfort, personalized care and safety [9].

One of the techniques currently under investigation for its potential is photoacoustic (PA) imaging $[10,11]$. PA mammography or PAMmography combines high intrinsic optical contrast of tumours with (US) resolution in detection, does not use ionizing radiation or contrast agents, and can be used without breast compression $[12,13]$. This makes it potentially an excellent technique for visualizing malignant breast abnormalities [14-18]. In PAMmography, the breast is illuminated 
with short pulses of near infrared (NIR) laser light. The light is absorbed by endogenous chromophores such as haemoglobin in the tissue. The absorbed energy leads, via the process of thermoelastic expansion, to a transient pressure wave that propagates through the tissue. The pressure wave can be detected with US detectors with the centre frequency and bandwidth appropriate for the specific application [11, 19]. Since US undergoes low scattering and attenuation, the resolution problems of pure optical imaging techniques due to high light scattering in tissue can largely be avoided [10, 11, 14]. The most prominent NIR absorber at malignant sites is haemoglobin, the concentration of which can be twice as high at malignant sites compared to healthy tissue [20, 21]. Furthermore, it is known from optical spectroscopy and magnetic resonance spectroscopy that the oxygen saturation and the concentrations of fat, water and collagen, all to some extent absorbing in the NIR regime, are altered at the location of malignancies [22-24]. This potentially gives NIR PA imaging the possibility of being sensitive for visualizing malignancies, and to be specific through use of spectroscopy methods [25, 26].

Over the years, prototype PA mammography systems have been investigated in early clinical feasibility studies. However, the amount of clinical data available remains limited $[15,16$, 27-29]. We developed the Twente Photoacoustic Mammoscope or PAM [30, 31], which can image a region of interest (ROI) in the breast in forward mode using 1 , $064 \mathrm{~nm}$ light excitation in combination with a 2D detector array comprising 588 elements. We showed that PAM can visualize breast cancer with high imaging contrast [28, 29], and that this contrast appears to be independent of the mammographically estimated breast density. However, these first studies were performed in small patient populations, and, due to technical constraints, the measurements were performed in a small ROI $\left(40 \times 40 \mathrm{~mm}^{2}\right.$ with measuring time of $25 \mathrm{~min}$ ). The small ROI led to positioning difficulties, which constrained us to measure a selection of palpable masses measuring between 1 and $3 \mathrm{~cm}$ in size [28]. Although these first measurements showed the potential of PA mammography for the visualization of malignancies, the small study population, poor variability in included lesions and small ROI measurements made it impossible to relate the photoacoustic lesion visibility and appearance to patient demographics and lesion characteristics. This information is required for further improvements to the performance of the technique.

In this work, we use an upgraded prototype clinical system, which enables us to measure a larger field of view (FOV; $90 \times 80 \mathrm{~mm}^{2}$ in scan duration of $10 \mathrm{~min}$ ). This provides the opportunity to investigate a wider variety of tumours and to obtain better resolution than previously. The standardized large FOV measurements allow for better comparisons with $\mathrm{X}$-ray mammography and magnetic resonance imaging (MRI). While a selection of the data has been presented previously
[32], here we look critically at all clinical data from malignancies obtained with the improved version of PAM. In addition to assessing the visibility of the lesion, the PA lesion features are compared to lesion and patient characteristics in order to further our knowledge of the performance and potential of the method, but above all to guide the research and developments towards future optimal breast imaging systems.

\section{Materials and methods}

\section{Clinical setting}

Clinical measurements were performed at the centre for breast care of Medisch Spectrum Twente (MST) in Oldenzaal, The Netherlands. The study protocol and informed consent procedure were approved by the hospital's review board. The study was registered in the Netherlands Trial Register (NTR) as TC 2945.

From April 2012 to May 2013, adult female patients arriving at the centre for breast care for conventional imaging of their breasts were asked to participate in the study. The focus was mainly on patients with suspect lesions following clinical investigation and conventional imaging. Since the penetration depth of PAM 1 is limited, mostly patients with lesions in the upper half of the breast were included.

All patients followed the normal diagnostic pathway: after clinical investigation and anamnesis, conventional imaging was performed. For x-ray imaging, cranio-caudal (CC) and medio-lateral-oblique (MLO) images of both breasts were made using the Lorad Selenia Full Field Digital Mammography System (Hologic, Bedford, MA, USA). Next, both breasts and axillae were investigated using the Philips iU22 US machine operating at $15 \mathrm{MHz}$ (Philips Medical Systems, Best, The Netherlands).

The suspect breast of a consenting patient was imaged using PAM (see below) before the core needle biopsy procedure. In cases when conventional imaging was not conclusive, a dynamic contrast enhanced MRI (DCE-MRI) was performed a few days to weeks post-biopsy. High-resolution anatomical T2 weighted images and dynamic T1 weighted images before and after gadolinium injection were acquired using a 1.5 or 3 Tesla MR system in combination with a 7 channel Sense Breast Coil (Philips Medical Systems).

The $\mathrm{X}$-ray, US and MR images were interpreted by a breast imaging radiologist. For all modalities, the radiologist graded the suspiciousness of the lesion according to the BI-RADS classification scale (Breast Imaging Reporting And Data System [33]), described the lesion appearance and location in the breast, and estimated the lesion size. Moreover, two radiologists independently graded the mammographic density of the breast in the CC x-ray mammograms using the BIRADS density scale $[33,34]$. This four-grade scale reflects 
the percentage of glandular tissue present in the breast. In case of markedly different opinions, a third radiologist could be solicited for an unbiased grading.

In the CC x-ray mammogram, a random area inside the malignancy and a random area outside, but adjacent to, the malignancy were chosen. The average intensity in both areas $\left(<I_{m}>\right.$ and $\left\langle I_{b}>\right.$ respectively) was used to calculate the contrast $\mathrm{C}$ as $\left\langle I_{m}\right\rangle\left|<_{I b}\right\rangle$. To reduce subjectivity in the choice of the specific areas, this procedure was repeated ten times and the average outcome was used to obtain $C_{x r a y}$ for the specific lesion. In case the lesion was occult on x-ray mammography, the contrast was defined as being 1 .

$C_{\text {xray }}=\frac{1}{10} \sum_{i}\left(\left\langle I_{m, i}\right\rangle /\left\langle I_{b, i}\right\rangle\right)$

\section{The Twente Photoacoustic Mammoscope}

Earlier published details of the instrument [30] as well as the study procedure [28] will be concisely repeated here. During the PAM investigation, the patient lies in prone position on the bed (Fig. 1) with her breast pendant through an aperture. The breast is immobilized by slight compression between a glass plate for laser illumination from the cranial side and the ultrasound detector array from the caudal side. This compression merely refers to placing the ultrasound detector on the breast and pushing it against the supporting glass plate to get an acoustic contact across the $85 \times 90$-mm detector surface for ultrasound detection. The static compression force applied is low so that tissue haemodynamics are not expected to be altered [35] and effects on the tumour contrast are negligible. After positioning, a photograph is made of the breast against the detector and the $\mathrm{CC}$ thickness of the breast under compression is measured using a caliper for the purpose of lesion depth estimation (see next section). By using the photograph of the breast in combination with the detector position, the $\mathrm{CC} \mathrm{x}$-ray images and PAM images can be merged retrospectively to provide an approximation of the co-localization of the identified lesions (see Fig. 2).

The breast is illuminated from the cranial side with pulsed $(10 \mathrm{~ns}, 10 \mathrm{~Hz})$ laser light at 1,064 nm from a Q-switched Nd:YAG laser (Continuum Surelite, San Jose, CA, USA). The size of the beam is approximately $35 \mathrm{~cm}^{2}$ and the beam is kept at a fixed position throughout the measurement. At the breast surface, the energy per pulse does not exceed $350 \mathrm{~mJ}$, giving rise to radiant exposure of $10 \mathrm{~mJ} / \mathrm{cm}^{2}$, which is well below the maximum permissible exposure (MPE) on the skin $\left(100 \mathrm{~mJ} / \mathrm{cm}^{2}\right)$ for the specifications of the laser light applied [36]. PA signals in the breast are detected at the caudal side of the breast by a $1 \mathrm{MHz}$ (130\% bandwidth) 2D US detector array (Lunar Corporation, General Electric, Madison, WI, USA) consisting of 588 elements in an almost circular configuration. Details of the detector are given elsewhere [30, 31]. Sequences of ten elements, spread over the entire detector area, are activated at a time. For all 588 elements, the average of 100 signals are read into the PC using two eight-channel digitizers (National Instruments, NI PXI 5105, 60 MS/s, $12-$ bit) controlled by a Labview program (National Instruments, Austin, TX, USA). This leads to a total measurement duration of $10 \mathrm{~min}$ for the complete detector covering an area on the breast of approximately $9 \times 8 \mathrm{~cm}^{2}$.

\section{Image reconstruction and analyses}

Reconstruction and analyses were performed using Matlab (R2011b, the Mathworks Company, Natick, MA, USA). Prior to reconstruction, the large signal from the breast surface was removed to have a more homogenous dynamic range in the PA volume. Signals were filtered with a band-pass Butterworth filter (cut-off frequencies $0.2 \mathrm{MHz}$ and 1.7 MHz) to remove the low frequency trend and high frequency noise. The PA volume was reconstructed from these processed signals using an acoustic back-projection algorithm, assuming a homogenous speed of sound of 1,540 m/s
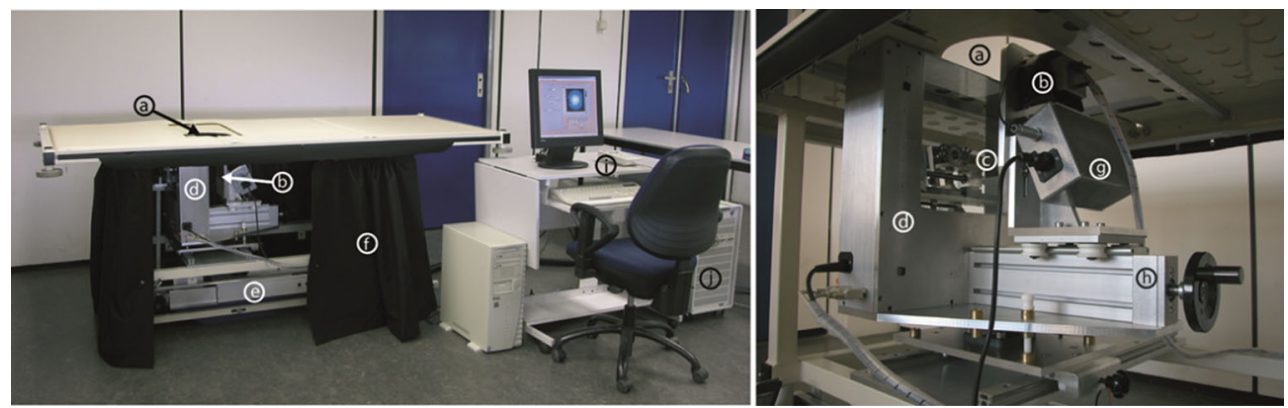

Fig. 1 The Twente Photoacoustic Mammoscope. (a) Aperture for breast insertion. (b) Ultrasound detector matrix. (c) Glass window. (d) Scanning system compartment. (e) Q-switched Nd-YAG laser operated at 1,064 nm with 10-ns pulses. (f) Laser safety curtain which is drawn around the instrument during the measurements. (g) Interface electronics between detector and computer. (h) Linear stage carrying detector matrix driven by hand wheel to apply mild compression to the breast. (i) Laser remote control unit. (j) Laser power supply. Image adapted from reference [29] with permission 


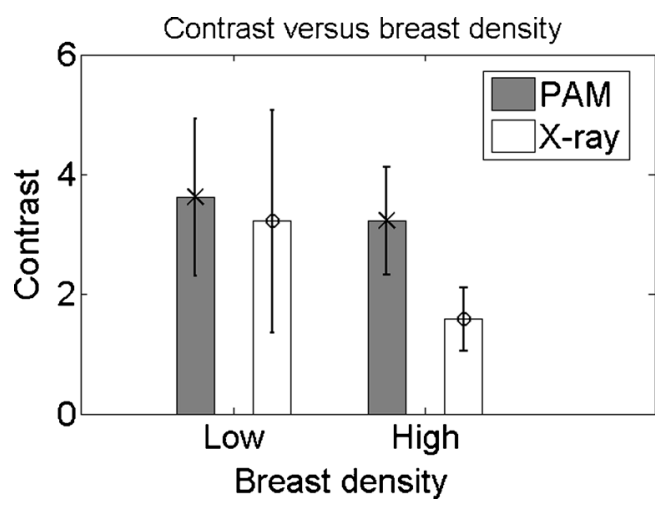

Fig. 2 Photoacoustic (PA) contrast versus breast density. There is no significant difference in PA contrast (grey, cross) between the low- and high-density breasts. There is a significant drop $(*)$ in contrast for $\mathrm{x}$-ray mammography (white, sphere) comparing the high-density to the lowdensity groups

in the medium. The inaccuracies that arise because of the assumption of this homogenous speed of sound are estimated to be minor with respect to the system's resolution (approximately $3 \mathrm{~mm}$ in both the axial and lateral directions [30,31]). After reconstruction, the separate profiles through the volume were demodulated and all voxel intensity values were scaled to the maximum intensity value within the total volume, giving a range between 0 and 255 .

The PA volume was investigated for the presence of highintensity areas. When high-intensity PA pixels were organized in a way reminiscent of lesion appearances from experiences in conventional imaging modalities, these structures were referred to as PA lesions. A subvolume was defined so as to include the depth (cranio-caudal) extension of the lesion. The maximum intensity projection (MIP) of the subvolume was overlaid on the $\mathrm{x}$-ray mammogram by using the position information obtained from the digital photograph.

In this way, lesion positions in the CC PA images could be compared to lesion positions in the $\mathrm{CC}$ x-ray images. In case of good co-localization, the lesion on PA images was assumed to represent the suspicious abnormality. The lesions were classified according to their appearance on the MIP of the subvolume as either showing 'mass (confined) appearance', 'non-mass (scattered) appearance' or 'ring appearance' (for further details, see below).

The depth of the lesion was calculated from the $\mathrm{z}$ (depth) position of the lesion's maximum intensity inside the PA volume and the measured breast thickness. A contour was manually drawn around the lesion on the MIP of the subvolume, using the information available from conventional imaging. This contour was used to measure the maximum dimension of the lesion in the $\mathrm{CC}$ projection and to calculate the average intensity inside the lesion $\left\langle I_{l}\right\rangle$ in this same MIP. Ten random areas outside but close to the lesion were chosen. For each area, the average intensity was calculated to obtain $\left\langle I_{b, i}>\right.$ and to calculate the contrast $\mathrm{Ci}$ of the lesion as $C_{i}=\left\langle I_{l}\right\rangle \mid\left\langle I_{b, i}\right\rangle$. To reduce subjectivity in the choice of the specific background area, the average of the ten calculated contrasts was used to obtain $C_{\text {pam }}$.

$C_{\text {pam }}=\frac{1}{10} \sum_{i}\left(\left\langle I_{l}\right\rangle /\left\langle I_{b, i}\right\rangle\right)$

The estimated size of the lesion in PA images was compared to the true lesion size defined histopathologically. For each modality (PAM, x-ray, US and MRI) The percentage (absolute) size deviation from the gold standard (histopathology) was calculated as:

$$
\left(\mid \text { Size }_{\text {Histo }}-\text { Size }_{\text {Mod }} \mid /{ }_{\text {Size }}{ }_{\text {Histo }}\right) \times 100 \%
$$

in which SizeMod is the measured maximum diameter of the lesion on images from the specific modality and SizeHisto is the maximum diameter of the lesion measured by the pathologist in the post-surgical specimen. If lesions were not visible, but correctly positioned in the measurement area, the size was defined to be $0 \mathrm{~mm}$ for that specific modality. Further, for all imaging modalities (x-ray, US, MRI and PAM) the correlation coefficient between the measured lesion sizes and the true lesion sizes was calculated.

Per BI-RADS breast density group, $\left\langle C_{\text {pam }}\right\rangle$ and $\left\langle C_{x r a y}\right\rangle$ were calculated and compared to have an indication of the influence of breast density on the relative performances of PAM and x-ray mammography. Both for PA and $\mathrm{x}$-ray lesions, the density groups were tested for significant differences in their mean contrast by using a (one-sided) two-sample Student's t-test with a significance level of $5 \%$.

\section{Other clinical information obtained}

Following core needle biopsy, all tissue specimens were investigated for the presence and type of malignancy at the Laboratory for Pathology East Netherlands (LabPON). Following surgery (mastectomy or breastconserving therapy), pathology was performed to determine the status of the resection margins, the type (according to WHO criteria) and size of the lesion, the grade of the lesion on the Bloom-Richardson scale [37], the presence of in situ components outside the lesion and the hormonal status of the tumour. All this information, except the status of the resection margins, was compared to the PA lesion visibility and appearance.

At the end of the PA measurement, patients were asked to fill a short questionnaire regarding their physical condition as well as their experience during the measurement. The PA lesion contrast can thus be compared to age, body mass index (BMI) and menopausal status of the patient. Pre-menopausal patients were 
asked to fill in the week of the cycle. From MRI it is known that results can be influenced by the hormonal status of the patient [38], and since MRI and PAMmography are believed to largely rely on the same contrast mechanism [32, 39], namely vascularization, this factor should be investigated here as well. Further information from the questionnaire concerned the preference of the patient for either x-ray mammography or PAMmography.

\section{Results}

From April 2012 to May 2013, 43 patients volunteered to participate in the PAM study. Of these, two patients were not imaged because of inability to climb on/lay on the bed for $10 \mathrm{~min}$. For two other patients it appeared retrospectively (from information obtained from the digital images, x-ray mammograms, MR and US images) that the lesion was not favourably positioned in front of the detector. Of the remaining 39 patient measurements, five were on cysts, two were on fibroadenoma, one was on a chronic active inflammation and 31 were on malignancies. The results for the measurements on malignancies are presented here; the results from the cyst measurements are published elsewhere [40]. Moreover, in a subset of the 43 patients from this study, a specific comparison with MRI and vascular stained histopathology slides was made in order to get more information on the relationship between the photoacoustic image pattern and the tumour vascularity [32]. The number of fibroadenomas measured was too low and the two lesions were too different in appearance and size ( $\sim 10 \mathrm{~mm}$ vs. $>60 \mathrm{~mm})$ on conventional imaging to analyze and compare their PA appearances.

\section{General photoacoustic (PA) results}

In 30 patients, the malignancies could be visualized with high certainty. This was confirmed by a high degree of colocalization between the lesions in X-ray and PA images. In the few cases where there was not good co-localization, the shift of the lesion in PA images compared to the expected location from the CC x-ray image could be related to an intended or unintended tilting of the breast during the PA investigation. In one patient (P60), the lesion could not be visualized at the expected position in the PA images. Based on data from x-ray mammography and MRI, mispositioning could neither be excluded nor fully ascertained.

\section{PA lesion characteristics}

Table 1 summarizes the results from PA imaging in comparison to the lesion type and size. The types of malignancies (25 infiltrating ductal carcinoma (IDC), four infiltrating lobular carcinoma (ILC) and/or lobular carcinoma in situ (LCIS), and two other/non-defined) measured in this study represent those of the normal breast cancer population [41-43]. Further patient characteristics are specified in Table 3.

The 32 detected lesions were classified according to their appearance as:

- 'Mass or confined appearance', defined as a confined region of high intensity, which can be either regular or irregular in shape and homogenous or heterogeneous in intensity.

- 'Non-mass or scattered appearance', defined as an area which is completely different from the surrounding healthy tissue by the presence of multiple moderate to high intensity foci.

- 'Ring appearance', defined as one or multiple regions of high intensity enclosing an area with low intensity.

Most lesions were of the mass-type, as in the first phase of the clinical study [28]. However, the lesions are much less smooth and show more heterogeneity in the current study. More details are discussed in [32].

Although the only lesion which was not visible in the PA images was of the less common ILC type, the variation in PA appearance cannot directly be related to the tumour type: There is large variation within the group of lesions of the same type (see for example Fig. 4c and f), while lesions of a completely different type can have a quite similar appearance (Fig. $4 \mathrm{c}$ and 1). Moreover, the number of non-IDC lesions (five) is too low with respect to the IDC group to make a reliable comparison between the different lesion types.

Representative PA images can be found in Fig. 4.

\section{PA lesion characteristics in relation to conventional imaging findings}

In Table 2 the PA lesion characteristics are compared to the lesion characteristics in x-ray mammography, US and MR images. Three lesions (P36_3, P38 and P44) were occult in $\mathrm{X}$-ray mammography images, of which one was also not observed in US images (P36_3). All these lesions could be identified with high imaging contrast in the PA images. The position of the lesion was verified by the position information from palpation and US imaging. For patient P36, at the time of the PAM investigation only the existence of two lesions was known; however, three lesions were observed in the PA images. The post-surgical histopathology report showed the existence of a third IDC at a location which co-localized with the third lesion in the PA images.

Figure 2 shows the breast density versus contrast for both $\mathrm{x}$-ray mammography and PAMmography. The final result of the density assessment (Table 2, column 2) by the three radiologists is used to define the density groups for this analysis 
Table 1 Results from photoacoustic imaging

\begin{tabular}{|c|c|c|c|c|c|c|c|}
\hline $\mathrm{P}^{1}$ & $\begin{array}{l}\text { Fig. } \\
\text { Nr. }\end{array}$ & Lesion type & Lesion $\operatorname{size}^{2}(\mathrm{~mm})$ & $\begin{array}{l}\text { PA } \\
\text { lesion appearance }\end{array}$ & $\begin{array}{l}\text { PA } \\
\text { contrast }\end{array}$ & PA size $(\mathrm{mm})$ & $\begin{array}{l}\text { Co-localization } \\
\text { x-ray/MRI }\end{array}$ \\
\hline 32 & \multirow{11}{*}{$4 a-c$} & IDC & 12 & Mass & 2.6 & 16 & Good \\
\hline 35 & & IDC & 26 & Mass & 2.7 & 18 & Good \\
\hline 36 & & $\operatorname{IDC}(3 \times)$ & $6,6,20$ & Mass $(3 \times)$ & $4.2,3.1,3.3$ & $8,8,11$ & Good \\
\hline 37 & & IDC & 28 & Ring & 2.2 & 31 & Good \\
\hline 38 & & IDC & 19 & Mass & 5.1 & 19 & Good \\
\hline 39 & & IDC & 63 & Non-mass & 3.6 & 41 & Reasonable \\
\hline 40 & & ILC & un & Non-mass & 3.7 & 45 & Poor $^{3}$ \\
\hline 42 & & IDC & un & Mass & 3.9 & 28 & Good \\
\hline 43 & & IDC & 15 & Mass & 2.9 & 16 & Good \\
\hline 44 & & IDC & 25 & Mass & 3.7 & 38 & Not defined \\
\hline 45 & & Adenotype & un & Non-mass & 3.2 & 61 & Reasonable $^{3}$ \\
\hline 47 & \multirow[t]{7}{*}{$4 d-f$} & IDC & 24 & Ring & 5.5 & 24 & Good \\
\hline $49^{4}$ & & LCIS & 40 & Non-mass & 3.9 & 54 & Good \\
\hline 52 & & IDC & un & Non-mass & 2.7 & 53 & Good \\
\hline 53 & & IDC & un & Ring & 3.3 & 46 & Good \\
\hline 54 & & IDC & 22 & Mass & 6.4 & 18 & Reasonable $^{5}$ \\
\hline 55 & & IDC & 34 & Mass & 2.8 & 29 & Good \\
\hline 56 & & IDC & 22 & Mass & 3.4 & 11 & Reasonable-Poor ${ }^{5}$ \\
\hline 58 & \multirow[t]{7}{*}{$4 j-l$} & ILC & 22 & Mass & 2.3 & 19 & Good \\
\hline 59 & & IDC & 28 & Non-mass & 3.5 & 29 & Reasonable \\
\hline 60 & & ILC & 22 & Not visible & $1.0^{6}$ & 0 & n.a. \\
\hline 61 & & IDC & un & Mass, atypical & 4.3 & 21 & Good \\
\hline 62 & & IDC & 18 & Mass & 2.6 & 15 & Good \\
\hline 63 & & IDC & un & Mass & 7.0 & 13 & Poor $^{5}$ \\
\hline 65 & & IDC & 18 & Mass & 2.6 & 13 & Good \\
\hline 66 & \multirow[t]{6}{*}{$4 g-i$} & $\mathrm{MC}$ & 15 & Mass & 4.0 & 15 & Good \\
\hline 67 & & IDC & 13 & Mass & 5.3 & 16 & Good \\
\hline 68 & & IDC & 14 & Mass & 2.9 & 10 & Good \\
\hline 70 & & IDC & 19 & Mass & 3.0 & 23 & Good \\
\hline 72 & & IDC & un & Non-mass & 2.8 & 44 & Good \\
\hline 73 & & IDC & 25 & Multifocal mass & 2.9 & 42 & Reasonable $^{5}$ \\
\hline
\end{tabular}

${ }^{1}$ Internal numbering system

${ }^{2}$ The size of the lesion is estimated from histopathology post-surgery. If patients did not undergo surgery in Medisch Spectrum Twente (MST), the size is indicated as 'unknown' (un)

${ }^{3}$ The size of the lesion and/or the high breast density made a comparison between PA and x-ray lesion localization difficult

${ }^{4}$ An adenotype metastasis was found in one of the lymph nodes; however, the post-mastectomy specimen only revealed LCIS in the breast. The metastasis proves that there should have been an invasive component

${ }^{5}$ For these patients, the non-perfect co-localization could be attributed to the significant breast tilting during the PA measurement;

${ }^{6}$ The lesions with a contrast of ' 1 ' for PA mammography were not visible in the PA imaging volume

$P A$ photoacoustic, $P$ patient number, MRI magnetic resonance imaging, IDC infiltrating ductal carcinoma, ILC infiltrating lobular carcinoma, $L C I S$ lobular carcinoma in situ, $M C$ mucinous carcinoma, n.a. not applicable, un unknown

(this will be further referred to as 'complete assessment'). Here, a two-scale classification is given (low: BI-RADS density 1, 2; high: BI-RADS density 3, 4), because this is proven to be more reliable and reproducible than the four-scale classification [34]. For both the individual assessment of the two radiologists and the complete assessment, there was no significant difference in average PA contrast between the high- and low-density groups, while the contrast of x-ray mammography is significantly lower for the high-density group as compared to the low-density group. Examples of lesions that are visible with high-imaging contrast in highly dense breasts can be found in the supplementary information. 
Table 2 Results from conventional imaging

\begin{tabular}{|c|c|c|c|c|c|c|c|}
\hline $\mathrm{P}^{1}$ & $\begin{array}{l}\text { BI-RADS } \\
\text { density }\end{array}$ & $\mathrm{x}$-ray contrast & PA contrast & $\begin{array}{l}\text { Size x-ray }(\mathrm{mm}) \\
(\% \text { deviation })^{2}\end{array}$ & $\begin{array}{l}\text { Size US }(\mathrm{mm}) \\
(\% \text { deviation })^{2}\end{array}$ & $\begin{array}{l}\text { Size MRI (mm) } \\
(\% \text { deviation })^{2}\end{array}$ & $\begin{array}{l}\text { Size PA }(\mathrm{mm}) \\
(\% \text { deviation })\end{array}$ \\
\hline 32 & 1 & 2.4 & 2.6 & $12(0 \%)$ & $11(-8 \%)$ & & $16(+33 \%)$ \\
\hline 35 & 1 & 2.3 & 2.7 & $24(-8 \%)$ & $25(-4 \%)$ & & $18(-31 \%)$ \\
\hline 36 & 2 & $3.1,2.0,1.0^{3}$ & $4.2,3.1,3.3$ & $6,10,0(0,+66,-100 \%)$ & $6,8,0(0,+33,-100 \%)$ & & $\begin{array}{l}8,8,11 \\
\quad(+33,+33,-45 \%)\end{array}$ \\
\hline 37 & 3 & 2.2 & 2.2 & $35(+25 \%$ & $36(+29 \%)$ & & $31(+11 \%)$ \\
\hline 38 & 3 & 1.0 & 5.1 & $0(-100 \%)$ & $21(+11 \%)$ & $22(+16 \%)$ & $19(0 \%)$ \\
\hline 39 & 2 & 5.6 & 3.6 & $50(-21 \%)$ & $50(-21 \%)$ & & $41(-35 \%)$ \\
\hline $40^{4}$ & 4 & $\mathrm{np}^{5}$ & 3.7 & np & $\mathrm{np}$ & np & 45 \\
\hline $42^{4}$ & 2 & 1.5 & 3.9 & 30 & np & & 28 \\
\hline 43 & 1 & 2.8 & 2.9 & $15(0 \%)$ & $15(0 \%)$ & & $16(+7 \%)$ \\
\hline 44 & 2 & 1.0 & 3.7 & $0(-100 \%)$ & $30(+20 \%)$ & & $38(+52 \%)$ \\
\hline $45^{4}$ & 3 & $\mathrm{np}^{5}$ & 3.2 & $\mathrm{np}$ & $\mathrm{np}$ & & 61 \\
\hline 47 & 2 & 6.6 & 5.5 & $25(+4 \%)$ & $29(+21 \%)$ & & $24(0 \%)$ \\
\hline $49^{6}$ & 2 & $\mathrm{np}^{5}$ & 3.9 & 15 & 0 & $20-50$ & 54 \\
\hline $52^{4}$ & 3 & 1.3 & 2.7 & 60 & 42 & 70 & 53 \\
\hline $53^{4}$ & 2 & 6.6 & 3.3 & 65 & 50 & & 46 \\
\hline 54 & 1 & 2.0 & 6.4 & $18(-18 \%)$ & $13(-41 \%)$ & & $18(-18 \%)$ \\
\hline 55 & 1 & 6.8 & 2.8 & $20(-41 \%)$ & $23(-32 \%)$ & $50(+47 \%)$ & $29(-15 \%)$ \\
\hline 56 & 3 & 1.3 & 3.4 & $n p^{3}$ & $34(+54 \%)$ & $32(+45 \%)$ & $11(-50 \%)$ \\
\hline 58 & 1 & 1.8 & 2.3 & $20(-9 \%)$ & $25(+14 \%)$ & $55(+150 \%$ & $19(-14 \%)$ \\
\hline 59 & 2 & 2.3 & 3.5 & $35(+25 \%)$ & $30(+7 \%)$ & & $29(+4 \%)$ \\
\hline 60 & 1 & 3.5 & 1.0 & $\mathrm{np}^{5}$ & $25(+14 \%)$ & $67(+204 \%)$ & $0(-100 \%)$ \\
\hline $61^{4}$ & 1 & 6.4 & 4.3 & 16 & 10 & 20 & 21 \\
\hline 62 & 3 & 1.4 & 2.6 & $20(+11 \%)$ & $20(+11 \%)$ & $19(+6 \%)$ & $15(-17 \%)$ \\
\hline $63^{4}$ & 1 & 2.7 & 7.0 & 22 & 21 & & 13 \\
\hline 65 & 2 & $1.0^{7}$ & 2.6 & $14(-22 \%)$ & $18(0 \%)$ & & $13(-28 \%)$ \\
\hline 66 & 2 & 3.0 & 4.0 & $20(+33 \%)$ & $16(+7 \%)$ & & $15(0 \%)$ \\
\hline 67 & 1 & 3.2 & 5.3 & $18(+38 \%)$ & $13(0 \%)$ & $15(+15 \%)$ & $16(+23 \%)$ \\
\hline 68 & 3 & 2.3 & 2.9 & $15(+7 \%)$ & $15(+7 \%)$ & & $10(-29 \%)$ \\
\hline 70 & 2 & 2.0 & 3.0 & $25(+32 \%)$ & $20(+5 \%)$ & $30(+58 \%)$ & $23(+21 \%)$ \\
\hline $72^{4}$ & 2 & 4.3 & 2.8 & 35 & 36 & 50 & 44 \\
\hline 73 & 2 & 3.4 & 2.9 & $49(+96 \%)$ & $26(+4 \%)$ & $35(+40 \%)$ & $42(+68 \%)$ \\
\hline
\end{tabular}

${ }^{1}$ Internal numbering system

${ }^{2}$ The percentage size deviation is calculated according to Formula 3. For lesions which were correctly positioned, but invisible, the lesion size was defined as $0 \mathrm{~mm}(100 \%$ size deviation)

${ }^{3}$ Lesions with a contrast of ' 1 ' for either $x$-ray mammography or PA imaging, were occult on the specific images

${ }^{4}$ These patients did not go for surgery at Medisch Spectrum Twente (MST), mostly because of the treatment by neoadjuvant chemotherapy. Therefore post-surgical histopathological sizes could not be obtained

${ }^{5}$ In these patients, the contrast and/or size of the lesion could not fully be estimated on conventional imaging, because of the appearance of architectural distortion rather than mass

${ }^{6}$ In this patient, an adenotype metastasis was found in one of the lymph nodes; however, the post-mastectomy specimen only revealed lobular carcinoma in situ in this breast. The metastasis proves that there should have been an invasive component. The size given here is the one of the in situ component. These are not taken into account in the estimation of the average size over- or underestimation for the different imaging modalities

${ }^{7}$ The lesion was visible in $\mathrm{x}$-ray mammography as a radio-opaque abnormality of approximately $14 \mathrm{~mm}$, associated with microcalcifications, hence the contrast was approaching ' 1 '

$P A$ photoacoustic, $P$ patient number, $M R I$ magnetic resonance imaging, US ultrasound, n.a. not applicable, n.p. not possible, un unknown

In this study, the average size deviations for $\mathrm{x}$-ray mammography, ultrasound and MRI were: $34 \%, 18 \%$ and $65 \%$, respectively. The average size deviation for PAMmography was $28 \%$. 
Table 3 Patient and lesion characteristics

\begin{tabular}{|c|c|c|c|c|c|c|c|}
\hline $\mathrm{P}^{1}$ & Age (y) & BMI & $\begin{array}{l}\text { Hormonal } \\
\text { status (week) }\end{array}$ & $\begin{array}{l}\text { Type and grade } \\
\text { of malignancy }\end{array}$ & DCIS (yes/no) & $\begin{array}{l}\text { Receptor status } \\
(\text { Er, Pr, Her2Neu) }\end{array}$ & Size of lesion ${ }^{2}(\mathrm{~mm})$ \\
\hline 32 & 54 & 28.7 & Post & IDC, grade 1 & No & ++- & 12 \\
\hline 35 & 79 & 35.6 & Post & IDC, grade 1 & No & ++- & 26 \\
\hline $36(3 x)$ & 56 & 27.5 & Post & IDC, grade $1(3 \times)$ & Yes & $++-(3 \times)$ & $6,6,20$ \\
\hline 37 & 61 & 25.9 & Post & IDC, grade 2 & No & +++ & 28 \\
\hline 38 & 44 & 26.0 & Pre (3) & IDC, grade 3 & Yes & - & 19 \\
\hline 39 & 67 & 33.2 & Post & IDC, grade 3 & No & ++- & 63 \\
\hline 40 & 47 & 26.2 & Pre (2) & ILC & n.a. & +++ & $\mathrm{un}^{3,4}$ \\
\hline 42 & 91 & 20.0 & Post & IDC & un & ++- & $\sim 30^{2}$ \\
\hline 43 & 83 & 25.0 & Post & IDC, grade 2 & Yes & ++- & 15 \\
\hline 44 & 55 & 24.5 & Post & IDC, grade 3 & Yes & + & 25 \\
\hline $45^{5}$ & 51 & un & Post & Adenotype & un & un & $\mathrm{un}^{3,4}$ \\
\hline 47 & 69 & 22.5 & Post & IDC, grade 2 & No & ++- & 24 \\
\hline 49 & 64 & 29.0 & Post & LCIS & n.a. & +- & $>40$ \\
\hline 52 & 44 & un & un & IDC & un & ++ un & $>60^{3}$ \\
\hline 53 & 72 & 21.3 & Post & IDC & un & + & $>60^{3}$ \\
\hline 54 & 70 & 30.1 & Post & IDC, grade 1 & No & ++- & 22 \\
\hline 55 & 63 & 24.8 & Post & IDC, grade 2 & No & + & 34 \\
\hline 56 & 62 & 26.9 & Post & IDC, grade 3 & No & +++ & 22 \\
\hline 58 & 65 & 27.7 & Post & ILC, grade 2 & n.a. & ++- & 22 \\
\hline 59 & 79 & 27.1 & Post & IDC, grade 3 & No & ++- & 28 \\
\hline 60 & 66 & 23.1 & Post & ILC, grade 1 & n.a. & ++- & 22 \\
\hline 61 & 74 & 41.4 & Post & IDC & un & ++- & $22^{3}$ \\
\hline 62 & 32 & 18.6 & Pre (1) & IDC, grade 2 & No & ++- & 18 \\
\hline 63 & 79 & 24.1 & Post & IDC & Un & ++- & $22^{3}$ \\
\hline 65 & 78 & un & Post & IDC, grade 1 & Yes & -+ & 18 \\
\hline 66 & 83 & 26.6 & Post & MC, grade 2 & Yes & ++- & 15 \\
\hline 67 & 73 & 23.8 & Post & IDC, grade 2 & Yes & ++- & 13 \\
\hline 68 & 63 & 25.5 & Post & IDC, grade 2 & Yes & ++- & 14 \\
\hline 70 & 63 & 29.8 & Post & IDC, grade 3 & No & ++- & 19 \\
\hline 72 & 61 & 24.3 & Post & IDC & un & ++- & $35^{3}$ \\
\hline 73 & 50 & 28.2 & Post & IDC, grade 3 & No & ++- & $25+5^{6}$ \\
\hline
\end{tabular}

${ }^{1}$ Internal numbering system

${ }^{2}$ The size of the lesion is estimated from histopathology post-surgery or conventional imaging

${ }^{3}$ These patients did not undergo surgery in Medisch Spectrum Twente (MST), therefore the lesion size, grade and presence of DCIS are not available. If possible the lesion size (last column) was estimated from conventional imaging

${ }^{4}$ In these patients, the size of the lesion could not be estimated from conventional imaging, because of the large extension in the breast and the appearance of architectural distortion rather than mass

${ }^{5}$ In this patient the biopsy of the lesion was inconclusive, but biopsies lymph node biopsies showed metastases from an adenotype carcinoma. The patient left to go to a different hospital for further diagnosis and treatment and was lost for follow-up

${ }^{6}$ In this patient, in addition to the $25 \mathrm{~mm}$ abnormality, a second, 5-mm focus of the same type of malignancy was found in the histopathology specimen. There were no invasive or in situ components present in between these lesions

$P$ patient number, BMI body mass index, DCIS ductal carcinoma in situ, IDC infiltrating ductal carcinoma, ILC infiltrating lobular carcinoma, $L C I S$ lobular carcinoma in situ, $M C$ mucinous carcinoma, un unknown, n.a. not applicable

\section{PA appearance versus patient and lesion characteristics}

Table 3 consolidates the patient and lesion characteristics for the 31 measured patients. No significant differences can be observed in PA contrast for the different hormonal receptor status groups. Figure 3 shows the average PA and $\mathrm{x}$-ray contrast for the three different tumour grades. There is no significant difference in PA contrast between any of the different groups. 


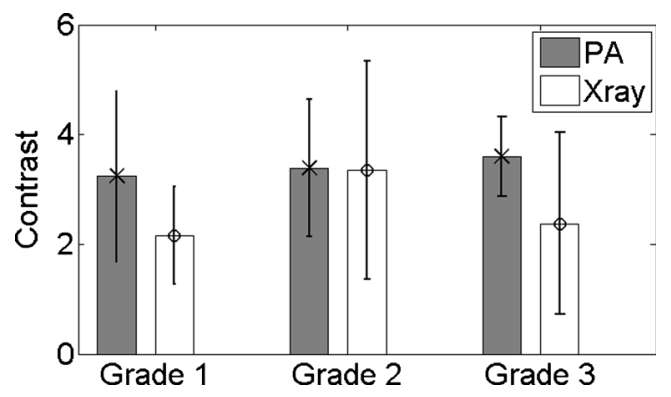

Fig. 3 Tumour grade versus contrast on photoacoustic (PA) images. There is no significant difference between the groups

The size deviation from the gold standard could not be related to the depth of the lesion, the hormonal receptor status or the presence of DCIS components outside the invasive malignancy.

Further, the average PA contrast was compared to the patients' BMI. Neither a trend nor a significant difference can be observed for the different BMI categories. This was also the case for the PA contrast versus the patient's age. In this study, only three pre-menopausal patients were measured, which were all in a different week of their menstrual cycle. The influence of the hormonal status on the PA results could therefore not be investigated.

\section{Specific representative PA mammography results}

Figure 4 shows the comparison between x-ray mammography and PA imaging for four representative patients in various overlays and projections.

In Fig. $4 \mathrm{~h}$ an elongated structure seems to be connected to the lesion, which is not that prominent in the x-ray images (Fig. 4g). A detailed look at the 3D volume reveals that the lobed and elongated structures are in fact separated and that the latter most likely represents a blood vessel. This can, however, not be verified, because of the lack of MR images for this patient. Such high-contrast areas, which were outside the region where the malignancy was expected and which could not always be attributed to known structures in the breast such as cysts or blood vessels, were present in approximately onethird of the patients investigated in this study.

\section{Patient experience}

Thirty patients completed the short questionnaire following the PAM investigation. Of these, 11 reported preferring PAM far more than x-ray mammography; 14 preferred PAM to $\mathrm{x}$-ray mammography; three considered the burden of $\mathrm{x}$-ray mammography to be equal to that of PAM; and two indicated a strong preference for $\mathrm{x}$-ray mammography over PAM. However, it must be noted here that patients were well aware of the fact that this system was a prototype and that it would not be applied in its current configuration in daily clinical practice outside a research setting.

\section{Discussion}

We reported here on the imaging results of clinical PA breast imaging. The results of 31 measurements on patients having one or more breast malignancies, obtained with a PA mammoscope which operates in planar configuration using $1,064 \mathrm{~nm}$ light [30,31], were presented and compared with patient demographics, lesion characteristics and results from conventional imaging. The most important observations are:

1. PAMmography can visualize breast malignancies with high imaging contrast;

2. The PA contrast of the lesions is independent of the mammographically estimated breast density, while there is a significant drop in lesion contrast in X-ray mammography for high-density breasts compared to low density breasts;

3. The PA lesion appearance and contrast could not be related to the lesion type, grade or hormonal status;

4. In a non-negligible number of patients, high contrast areas could also be observed in areas in the PA volume which could not be related to the location of the (proven) malignancy.

Tese four most important findings are discussed in more detail below and possibilities for future improvements are given.

\section{Visualizing breast malignancies}

In agreement with results from previous clinical studies [28, 29], this study proved that PA imaging as currently applied in our prototype planar mode imaging system using 1,064 nm can visualize breast malignancies with high imaging contrast. In some cases even malignancies that were occult on one of the conventional imaging modalities could be clearly visualized with reliable shape and size. This resulted in a visualization rate of 32 (of 33) malignancies in 31 patients in this study, while only one malignancy was occult in PA images.

The missed malignancy (P60) was an ILC, which presented as architectural distortion in $\mathrm{X}$-ray images and as a suspicious hypo-echoic area in US images. It is known that there are morphological differences between IDC (the most common measured malignancy in this study) and ILC, making the visualization of ILC in conventional imaging sometimes troublesome [42-44] and possibly also causing difficulties in PA visualization of ILC. However, two cases of ILC (P40 and P58) were visualized using PAM. Further, ILC is often associated with increased tumour vascularization as a consequence of angiogenic processes [45, 46], which caused the lesion of P60 to be visible as an enhancing mass in MR images. In fact, MRI has proven to be the most useful imaging modality in the pre-operative investigation of ILC [42, 47]. If the PA contrast 


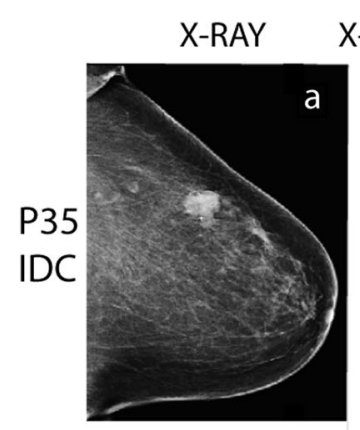

X-RAY+PHOTOACOUSTIC

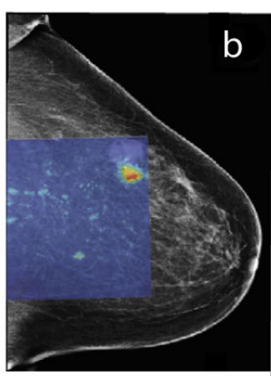

b

PHOTOACOUSTIC 3D
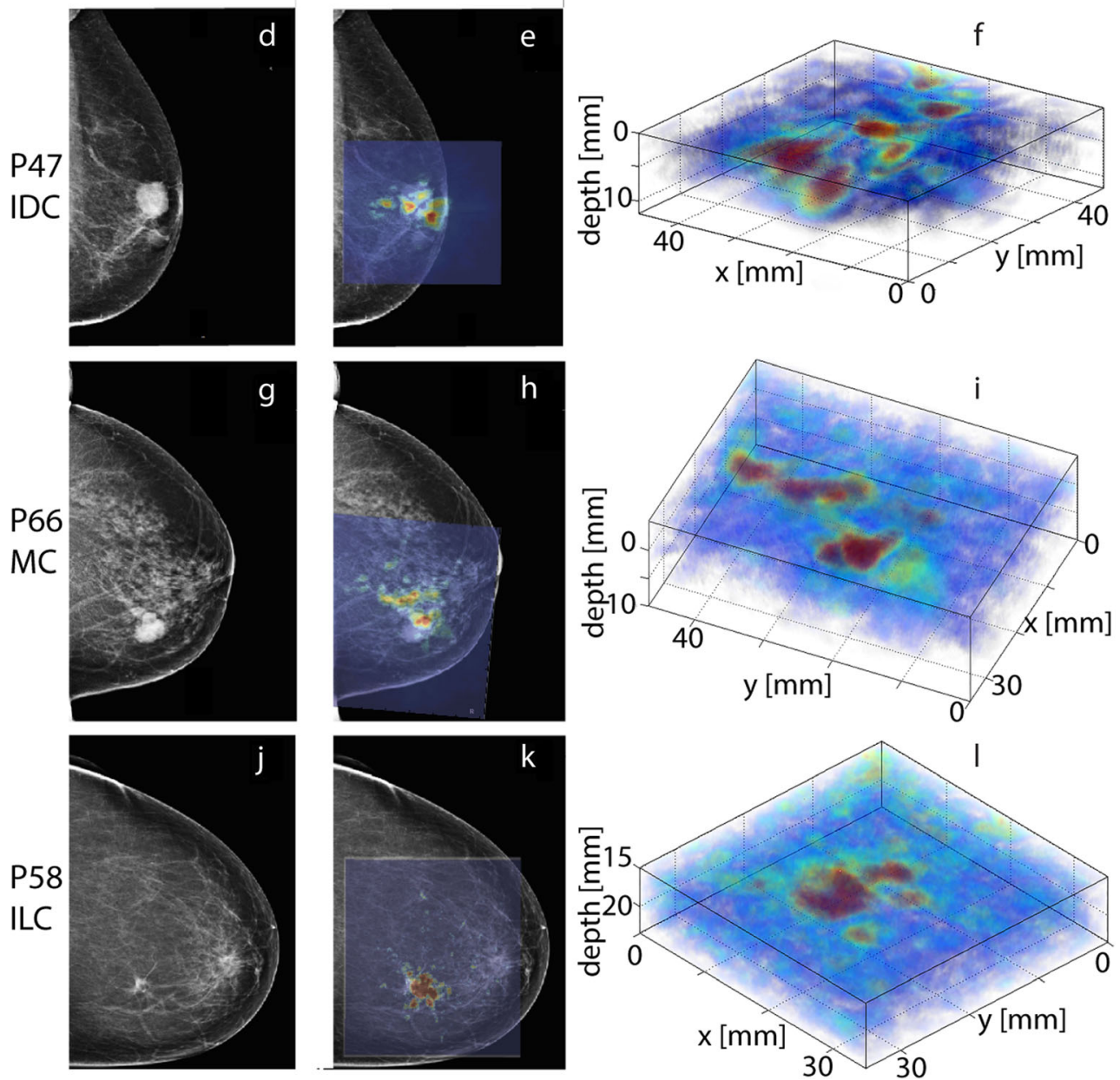

Fig. 4 Four representative photoacoustic imaging results. Left: Left cranio-caudal (CC) x-ray mammogram; middle: Left CC x-ray mammogram with maximum intensity projection (MIP) of the photoacoustic (PA) mammography-identified lesion overlaid; right: detailed 3D representation of the lesion of interest. (a-c) This infiltrating ductal carcinoma (IDC, grade 1) of $26 \mathrm{~mm}$ in a 79-year-old patient presents as a high-contrast mass appearance of $18 \mathrm{~mm}$ in the PA volume. The size underestimation is most likely the consequence of the poor positioning of the lesion at the edge of the detector. The shift in lesion location between the x-ray and PA images is most likely the consequence of the breast being slightly tilted following detector movement to immobilize the breast against the glass window prior to the measurement. (d-f) This infiltrating ductal carcinoma (IDC, grade 2) of $24 \mathrm{~mm}$ in a 69-year-old patient presents as a ring (diameter $24 \mathrm{~mm}$ ) in

is indeed mainly the consequence of the increased tumour vascularization, one would expect this lesion type to be visible

the PA volume. The ring appearance is hypothesized to be partly the consequence of a higher vascular density at the lesions border as compared to its centre [32]. Since x-ray mammography relies on a different contrast mechanism, this ring appearance does not necessarily appear in the x-ray images. (g-i) This mucinous carcinoma (MC, grade 2) of $15 \mathrm{~mm}$ detected in an 83-year-old patient presents as a lobed mass of $15 \mathrm{~mm}$ in the PA images. (j-l) This infiltrating lobular carcinoma (ILC, grade 2) of $22 \mathrm{~mm}$ in a 65 -year-old patient presents as an irregularly shaped mass of $19 \mathrm{~mm}$ in PA images. The location of the lesion in the PA volume can be perfectly co-localized with the location of the lesion on the x-ray mammogram. Even at depths of more than $20 \mathrm{~mm}$, the lesion can be visualized with good contrast and a rather reliable (although slightly underestimated) size

in the PA image as well. A further comparison between the digital photograph of the breast during the PAM investigation 
and the x-ray and MR images cannot exclude the possibility of mispositioning of the lesion in front of the detector. In any case, it is recommended that more patient measurements on ILC are performed.

A few aspects of the general results of the 31 visualized malignancies require further discussion. First, although previously all lesions were observed as homogenous and rather smooth masses, in the current study we see larger variations in PA lesion appearance. This higher variation can largely be explained by the higher variation in included patients and lesions: where we previously focused on small palpable masses, in this study non-palpable lesions, lesions with appearances of architectural distortion in $\mathrm{x}$-ray images and very large $(>60 \mathrm{~mm})$ and very small $(<10 \mathrm{~mm})$ lesions were also measured. Moreover, the increase in illuminating beam size and FOV leads to a more reliable reconstruction of the true lesion appearance. This might also explain the problems we still observe for the reconstruction of very large $(>40 \mathrm{~mm})$ lesions.

Second, in this study the PAM-estimated lesion sizes were both larger and smaller than the histopathologically defined lesion sizes, while previously the lesion size was always underestimated in the PA images [28]. The previous size underestimation was also most likely the consequence of the limited FOV with respect to the lesion size, a problem that is largely avoided in the current clinical study. Moreover, in the previous study, the lesions were defined by $-6 \mathrm{~dB}$ thresholds, while in this work a manual discrimination of 'abnormal' with respect to normal tissue was used. The latter is closer to the method that radiologists use for conventional imaging and leads to an average size deviation for PAM with respect to histopathology of $28 \%$ compared to size deviations of $34 \%, 18 \%$ and $65 \%$ for x-ray mammography, US and MRI, respectively. Therefore, PAM performs reasonably well compared to the other imaging modalities. However, nonnegligible under- and overestimations in lesion size are still observed, despite the fact that the lesions are defined with prior knowledge from conventional imaging. This size deviation is partly the consequence of the slight compression applied in the PAM set-up, causing the breast and lesions to deform. Moreover, in histopathological investigations (the gold standard) the largest dimension of the malignancy is measured, regardless of the orientation of the malignancy, while in PA images the largest dimension on a craniocaudal projection is taken. A more thorough analysis into the different chromophores that are contributing to the PA lesion contrast is required to truly relate the PA-defined size and appearance to the histopathologically-defined size and appearance.

\section{Contrast versus density}

As in our previous study [28], this work indicates that the PA contrast is independent of the mammographically estimated breast density. In the present study, the breast density was estimated in 31 cases by two (or three) radiologists, which is an improvement from the single observer in our 2012 study (ten patients). The third radiologist's judgement was used in 11 (of 31) cases and mostly occurred within and not between the low (BI-RADS density 1,2) or high (BI-RADS density 3 , 4) groups. Therefore, we used a two-grade scale to investigate the contrast dependence on breast density. Again, no significant differences can be observed in average PA contrast for the different density groups, while there is a significant drop in $\mathrm{x}$ ray contrast for the high-density breasts compared to the lowdensity breasts. Although the number of patients per density group is still limited, this repeated finding indicates that PA contrast at $1,064 \mathrm{~nm}$ is largely independent on the mammographically estimated breast density. This is most likely the consequence of the independence of the total blood volume and oxygen saturation on mammographic breast density [48]. In future multi-wavelength studies, the contrastdensity dependence should be investigated per wavelength since the water, fat and collagen content of high-density breasts are known to be different from that of lower density breasts [48].

\section{Contrast and appearance versus tumour type and grade}

A number of lesion and patient characteristics were investigated for their relationship to PA appearance, PA contrast and PA size. In the investigated population, the number of nonIDC was too low with respect to the number of IDC to compare the PA lesion appearances for the different tumour types. The same was the case for the number of pre-menopausal patients with respect to post-menopausal patients. No significant differences were found for the PA contrast versus BMI, PA contrast versus age, PA contrast versus hormone receptor status, PA contrast versus lesion depth or size deviation versus either lesion depth or the presence of in situ components.

The positive correlation between MVD and tumour grade in IDC [45, 49] led to the expectation of an increasing PA contrast with increasing tumour grade. This expectation makes it tempting to observe a subtle trend of increasing PA contrast with increasing tumour grade in Fig. 3. However, the none of the differences in PA contrast between the different tumour grades are significant. The main problem of the analyses performed here is the small number of patients per group, especially in cases where a lot of groups were compared, as for BMI or hormone receptor status. The presented results indicate that in future studies with larger numbers of patients, it will be useful to investigate the appearance of ILC with respect to IDC as well as the dependence of PA contrast on tumour grade. Moreover, the results further strengthen the hypothesis that PA imaging performs equally well in various population groups (age, BMI and breast density). 


\section{Sensitivity versus non-specificity}

The main conclusion of this work should be put into perspective. Using a priori information about the lesion location in the breast, for almost all patients abnormal or high intensity regions could be located and related to the presence of malignancy with great certainty. However, in a non-negligible number of patients high-contrast or abnormally structured areas were also observed at different locations in the PA volume, as illustrated in Fig. $4 \mathrm{~g}-\mathrm{i}$. In some cases, these high-contrast areas could be related to structures in the breast, like blood vessels, cysts or the nipple. For this, the lesion shape information as well as the information from the digital photograph and conventional imaging was used. However, there are also patients in whom, without prior knowledge about either the presence or the position of the malignancy, differentiating malignant from other high-intensity areas would have been very difficult using the current imaging system. Although we cannot give numbers for sensitivity and specificity for PAM due to the current study design, this observation indicates that currently the specificity of PAM is limited. This was to be expected from the use of a single wavelength. Although the wavelength used, 1,064 nm, is believed to be mostly absorbed by haemoglobin [19], other chromophores are also known to absorb this wavelength [19]. Moreover, haemoglobin is not a sole indicator of malignancy, but is also present in larger and smaller blood vessels in normal breast tissue. Therefore, in future PA imaging systems, better specificity can be achieved by the optimal choice of wavelengths. A wavelength combination that can for example differentiate the contribution of oxy-haemoglobin from that of deoxy-haemoglobin or the contribution of haemoglobin from that of water, can be very useful in this, since both the oxygen saturation as well as the water content are known to be altered at malignant sites [24, $50,51]$. The high visualization rate seems to indicate very good sensitivity of PA imaging for the detection of malignancies. However, true sensitivity and specificity numbers can only be determined in a blind study using an imaging system that can visualize the complete breast.

\section{Conclusions}

PAMmography can visualize breast malignancies with high imaging contrast using the Twente Photoacoustic Mammoscope, operating in planar mode and using one wavelength. All detected lesions could be co-localized with great certainty with the lesions on conventional imaging. The size estimation was comparable to conventional imaging techniques with an average $28 \%$ deviation from the gold standard. From a comfort point of view, by far most patients strongly preferred the PAM investigation to conventional $\mathrm{x}$-ray mammography.
The contrast at 1,064 $\mathrm{nm}$ was independent of the radiological breast density. All in all, the study confirms the high potential of PA mammography in breast care. However, the appearance of high-contrast areas outside the malignant region indicates that the specificity of the implementation with the use of a solitary wavelength is not optimal. The results further indicate that in future studies it is useful to investigate the appearance of ILC with respect to IDC as well as the dependence of PA contrast on tumour grade in a larger number of patients.

Acknowledgments The scientific guarantor of this publication is Srirang Manohar, PhD. (Associate Professor, University of Twente, The Netherlands.) Frank van den Engh, Wiendelt Steenbergen and Srirang Manohar declare relationships with the following company: PA Imaging BV.

The project was funded via the Rijksdienst voor Ondernemend Nederland (RVO.NL) Innovation-Oriented Research Programme (IOP) Photonic Devices under the HYMPACT Project (IPD083374). Other sources of support are: MIRA Institute for Biomedical Technology and Technical Medicine; and the VICI grant 10831 (Netherlands Technology Foundation STW) to W.S. No complex statistical methods were necessary for this paper. Institutional Review Board approval was obtained. The study protocol was approved by the Medisch Spectrum Twente Hospital's Review Board. The study has been registered in The Netherlands Trial Register (NTR) as TC 2945. Written informed consent was obtained from all patients in the study. The informed consent procedure was approved the Medisch Spectrum Twente Hospital's Review Board. Some study subjects of this cohort have been previously reported in Heijblom et al. Scientific Reports (2016), [32]. Methodology: retrospective, observational, performed at one institution.

Open Access This article is distributed under the terms of the Creative Commons Attribution-NonCommercial 4.0 International License (http:// creativecommons.org/licenses/by-nc/4.0/), which permits any noncommercial use, distribution, and reproduction in any medium, provided you give appropriate credit to the original author(s) and the source, provide a link to the Creative Commons license, and indicate if changes were made.

\section{References}

1. Siegel R, Naishadham D, Jemal A (2013) Cancer statistics, 2013. CA Cancer J Clin 63(1):11-30

2. DeSantis C, Siegel R, Bandi P, Jemal A (2011) Breast cancer statistics, 2011. CA-A Canc J Clin 61(6):409-418

3. Zografos G, Koulocheri D, Liakou P et al (2013) Novel technology of multimodal ultrasound tomography detects breast lesions. Eur Radiol 23(3):673-683

4. Sadigh G, Carlos RC, Neal CH, Dwamena BA (2012) Accuracy of quantitative ultrasound elastography for differentiation of malignant and benign breast abnormalities: a meta-analysis. Breast Cancer Res Treat 134(3):923-931

5. Jochelson MS, Dershaw DD, Sung JS et al (2013) Bilateral contrast-enhanced dual-energy digital mammography: feasibility and comparison with conventional digital mammography and MR imaging in women with known breast carcinoma. Radiology 266(3):743-751 
6. Linden HM, Dehdashti F (2013) Novel methods and tracers for breast cancer imaging. Semin Nucl Med 43(4):324-329

7. Grzegorczyk TM, Meaney PM, Kaufman PA, diFlorio-Alexander RM, Paulsen KD (2012) Fast 3-D tomographic microwave imaging for breast cancer detection. IEEE Trans Med Imag 31(8):15841592

8. Fantini S, Sassaroli A (2012) Near-infrared optical mammography for breast cancer detection with intrinsic contrast. Ann Biomed Eng 40(2):398-407

9. Drukteinis JS, Mooney BP, Flowers CI, Gatenby RA (2013) Beyond mammography: new frontiers in breast cancer screening. Am J Med 126(6):472-479

10. Lutzweiler C, Razansky D (2013) Optoacoustic imaging and tomography: reconstruction approaches and outstanding challenges in image performance and quantification. Sensors 13(6):7345-7384

11. Wang LHV, Hu S (2012) Photoacoustic tomography: in vivo imaging from organelles to organs. Science 335(6075):1458-1462

12. Xia W, Steenbergen W, Manohar S (2014) Photoacoustic mammography: prospects and promises. Breast Canc Manag 3(5):387-390

13. Heijblom M, Steenbergen W, Manohar S (2015) Clinical photoacoustic breast imaging: the Twente experience. IEEE Pulse Spec Iss 6(3):42-46

14. Mallidi S, Luke GP, Emelianov S (2011) Photoacoustic imaging in cancer detection, diagnosis, and treatment guidance. Trends Biotechnol 29(5):213-221

15. Kruger RA, Lam RB, Reinecke DR, Del Rio SP, Doyle RP (2010) Photoacoustic angiography of the breast. Med Phys 37(11):60966100

16. Kitai T, Torii M, Sugie T et al (2014) Photoacoustic mammography: initial clinical results. Breast Cancer 21(2):146-153

17. Li X, Heldermon CD, Yao L, Xi L, Jiang H (2015) High resolution functional photoacoustic tomography of breast cancer. Med Phys 42(9):5321-5328

18. Kruger RA, Kuzmiak CM, Lam RB, Reinecke DR, Del Rio SP, Steed D (2013) Dedicated 3D photoacoustic breast imaging. Med Phys 40(11):113301

19. Beard P (2011) Biomedical photoacoustic imaging. Interfac Foc 1(4):602-631

20. Wang J, Pogue BW, Jiang SD, Paulsen KD (2010) Near-infrared tomography of breast cancer hemoglobin, water, lipid, and scattering using combined frequency domain and $\mathrm{cw}$ measurement. Opt Lett 35(1):82-84

21. Brown JQ, Wilke LG, Geradts J, Kennedy SA, Palmer GM, Ramanujam N (2009) Quantitative optical spectroscopy: a robust tool for direct measurement of breast cancer vascular oxygenation and total hemoglobin content in vivo. Cancer Res 69(7):2919-2926

22. Haddadin IS, McIntosh A, Meisamy S et al (2009) Metabolite quantification and high-field MRS in breast cancer. NMR Biomed 22(1):65-76

23. Chung SH, Cerussi AE, Klifa $\mathrm{C}$ et al (2008) In vivo water state measurements in breast cancer using broadband diffuse optical spectroscopy. Phys Med Biol 53(23):6713-6727

24. Cerussi A, Shah N, Hsiang D, Durkin A, Butler J, Tromberg BJ (2006) In vivo absorption, scattering, and physiologic properties of 58 malignant breast tumors determined by broadband diffuse optical spectroscopy. J Biomed Opt 11(4)

25. Ntziachristos V, Razansky D (2010) Molecular imaging by means of multispectral optoacoustic tomography (MSOT). Chem Rev 110(5):2783-2794

26. Taruttis A, van Dam GM, Ntziachristos V (2015) Mesoscopic and macroscopic optoacoustic imaging of cancer. Cancer Res 75(8): $1548-1559$

27. Ermilov SA, Khamapirad T, Conjusteau A et al. (2009) Laser optoacoustic imaging system for detection of breast cancer. J Biomed Opt 14(2):024007
28. Heijblom M, Piras D, Xia W et al (2012) Visualizing breast cancer using the Twente photoacoustic mammoscope: what do we learn from twelve new patient measurements? Opt Express 20(11): 11582-11597

29. Manohar S, Vaartjes SE, van Hespen JC et al (2007) Initial results of in vivo non-invasive cancer imaging in the human breast using near-infrared photoacoustics. Opt Express 15(19):12277-12285

30. Manohar S, Kharine A, van Hespen JCG, Steenbergen W, van Leeuwen TG (2005) The Twente Photoacoustic Mammoscope: system overview and performance. Phys Med Biol 50:2543-2557

31. Piras D, Xia WF, Steenbergen W, van Leeuwen TG, Manohar S (2010) Photoacoustic imaging of the breast using the twente photoacoustic Mammoscope: present status and future perspectives. IEEE J Select Topic Quant Electron 16(4):730-739

32. Heijblom M, Piras D, Brinkhuis M et al (2015) Photoacoustic image patterns of breast carcinoma and comparisons with Magnetic Resonance Imaging and vascular stained histopathology. Sci Rep 5: 11778

33. The ACR Breast Imaging Reporting and Data System (BIRADS). 2003 [cited 2013 July]; Available from: www.acr.org.

34. Ciatto S, Houssami N, Apruzzese A et al (2005) Categorizing breast mammographic density: intra- and interobserver reproducibility of BI-RADS density categories. Breast 14(4):269-275

35. Carp SA, Sajjadi AY, Wanyo CM et al (2013) Hemodynamic signature of breast cancer under fractional mammographic compression using a dynamic diffuse optical tomography system. Biomed Opt Expr 4(12):2911-2924

36. Commission, I E (Edition 1:1993 consolidated with amendments 1: 1997 and 2:2001) International standard, Safety of laser products, IEC 60825-1:1993+A1:1997+A2:2001, in Part 1: Equipment classification, requirements and user's guideInternational Electrotechnical Commission: Geneva, Switzerland

37. Bloom HJ, Richardson WW (1957) Histological grading and prognosis in breast cancer; a study of 1409 cases of which 359 have been followed for 15 years. Br J Cancer 11(3):359-377

38. Sardanelli F, Boetes C, Borisch B et al (2010) Magnetic resonance imaging of the breast: Recommendations from the EUSOMA working group. Eur J Cancer 46(8):1296-1316

39. Heijblom M, Klaase JM, van den Engh FM, van Leeuwen TG, Steenbergen W, Manohar S (2011) Imaging tumor vascularization for detection and diagnosis of breast cancer. Technol Cancer Res Treat 10(6):607-623

40. Heijblom M, Piras D, Maartens E et al (2013) Appearance of breast cysts in planar geometry photoacoustic mammography using 1064nm excitation. J Biomed Opt 18(12):126009

41. Li CI, Anderson BO, Daling JR, Moe RE (2003) Trends in incidence rates of invasive lobular and ductal breast carcinoma. JAMAJ Am Med Assoc 289(11):1421-1424

42. Lopez JK, Bassett LW (2009) Invasive lobular carcinoma of the breast: spectrum of mammographic, US, and MR imaging findings. Radiographics 29(1):165-176

43. Pestalozzi BC, Zahrieh D, Mallon E et al (2008) Distinct clinical and prognostic features of infiltrating lobular carcinoma of the breast: combined results of 15 International Breast Cancer Study Group clinical trials. J Clin Oncol 26(18):3006-3014

44. Cocquyt V, Van Belle S (2005) Lobular carcinoma in situ and invasive lobular cancer of the breast. Curr Opin Obstet Gynecol 17(1):55-60

45. Bolat F, Kayaselcuk F, Nursal TZ, Yagmurdur MC, Bal N, Demirhan B (2006) Microvessel density, VEGF expression, and tumor-associated macrophages in breast tumors: correlations with prognostic parameters. J Exp Clin Cancer Res 25(3):365-372

46. Vamesu $\mathrm{S}$ (2008) Angiogenesis and tumor histologic type in primary breast cancer patients: an analysis of 155 needle core biopsies. Rom J Morphol Embryol 49(2):181-188 
47. Boetes C, Veltman J, van Die L, Bult P, Wobbes T, Barentsz JO (2004) The role of MRI in invasive lobular carcinoma. Breast Cancer Res Treat 86(1):31-37

48. Taroni P, Pifferi A, Quarto G et al (2010) Noninvasive assessment of breast cancer risk using time-resolved diffuse optical spectroscopy. J Biomed Opt 15(6)

49. Valkovic T, Dobrila F, Melato M, Sasso F, Rizzardi C, Jonjic N (2002) Correlation between vascular endothelial growth factor, angiogenesis, and tumor-associated macrophages in invasive ductal breast carcinoma. Virchows Arch 440(6):583-588

50. Nachabé R, Evers DJ, Hendriks BHW et al (2011) Diagnosis of breast cancer using diffuse optical spectroscopy from 500 to $1600 \mathrm{~nm}$ : comparison of classification methods. J Biomed Opt 16(8):087010-1:12

51. van Veen RLP, Amelink A, Menke-Pluymers M, van der Pol C, Sterenborg HJCM (2005) Optical biopsy of breast tissue using differential path-length spectroscopy. Phys Med Biol 50(11):2573-2581 